\title{
Cholesterol and Lipid Rafts as Regulators of Signaling Through the EGF Receptor in Keratinocytes
}

\author{
Sylviane Lambert ${ }^{1}$, Robert Gniadecki ${ }^{2}$ and Yves Poumay ${ }^{*}, 1$ \\ ${ }^{I}$ Cell and Tissue Laboratory, URPHYM, University of Namur (FUNDP), Namur, Belgium \\ ${ }^{2}$ Department of Dermatology, University of Copenhagen, Bispebjerg Hospital, Copenhagen NV, Denmark
}

\begin{abstract}
The EGF receptor (HER1/EGFR) and its cognate ligands are of considerable interest in epidermal biology and pathology. HER1/EGFR is expressed throughout the entire epidermis and its signaling has been shown involved in the control of keratinocyte proliferation, adhesion, and differentiation. The signaling of HER1/EGFR can be modulated by the receptor's immediate environment, particularly by its localization or not in lipid rafts. These membrane domains are nowadays described as heterogeneous dynamic signaling platforms where lipid molecules, cholesterol especially, are highly ordered and where transmembrane proteins encounter a specialized environment. Cholesterol depletion has been used in order to disorganize lipid rafts in keratinocytes and induces activation of HER1/EGFR (without ligand addition), as well as downstream signaling. The activation of p38 MAPK obtained after cholesterol depletion is responsible for the induction of involucrin expression while keratin expression is suppressed. However in keratinocytes recovering from cholesterol depletion the activation of p38 MAPK induces a strong expression of the heparin-binding EGF-like growth factor, a ligand of HER1/EGFR. Lipid rafts could thus be membrane platforms that regulate cell signaling in keratinocytes in normal, but also pathological conditions.
\end{abstract}

\section{INTRODUCTION}

Growth factors of the epidermal growth factor (EGF) family demonstrate high potency in the control of epidermal keratinocytes in normal skin and in diseases. This control works through the EGF receptor (EGFR) also named Human EGF Receptor 1 (HER1), the integral protein that binds members of the EGF family on its extracellular moiety and exhibits ligand-induced tyrosine kinase activity in its cytoplasmic domain. The function of HER1/EGFR can be regulated by its molecular environment in the plasma membrane. In the present chapter, we will depict important functions played by HER1/EGFR in epidermal keratinocytes and how lipid rafts, specialized domains of the plasma membrane, influence HER1/EGFR functioning in general, but also in the epidermal context where lipid rafts can modulate the epidermal phenotype.

\section{IMPORTANCE OF THE EGF RECEPTOR IN THE PHYSIOLOGY OF KERATINOCYTES}

The growth factor receptors relay through the plasma membrane crucial information brought by the release of ligands in the cell surrounding, creating intracellular signals intended to regulate critical cellular functions. Among those receptors, the epidermal growth factor (EGF) receptor family of receptors is named the human EGF receptor (HER) family in the human species and its members result from the transcription of the different ErbB genes. The EGF receptor acts on major and disparate cellular functions such as cell

*Address correspondence to this author at the Cell \& Tissue Laboratory, URPHYM, University of Namur (FUNDP), 61, rue de Bruxelles, B-5000 Namur, Belgium; Tel: +32-81-724257; Fax: +32-81-724261;

E-mail: yves.poumay@fundp.ac.be proliferation, motility, adhesion and differentiation. For instance, EGF was named originally as epidermal due to its ability to stimulate proliferation of murine epidermal cells in the pioneering work of Cohen (for a historical review, see [1]). Consequently, there has been considerable interest for the HER family and for their ligands in the epidermal tissue as this system can control several epidermal functions. Thus, the members of the human EGF receptors family numbered HER1, HER2 and HER3, have been found expressed in human epidermal keratinocytes in vitro, as well as in vivo. In human skin, the HER1/EGFR is predominant and localized throughout the entire epidermis, although its expression is maximal in the basal layer, whereas HER2 and 3 are predominant in suprabasal layers [2-4]. No epidermal expression of HER4 has been reported yet.

The relevance of the HER family in keratinocytes is not only suggested by the expression of three members, it is also sustained by the numerous possible ligands expressed by keratinocytes themselves, including TGF $\alpha$, HB-EGF, or amphiregulin as ligands of HER1/EGFR [5-7], and also heregulins as ligands for HER3 [2,8]. It is of interest to notice that signaling events that depend on the activation of HER family members as homodimers or heterodimers are not redundant, illustrating by their diversity the significance of these receptors signaling pathways.

The following paragraphs briefly overview major cell functions that are under the control of HER1/EGFR signaling in epidermal keratinocytes.

\subsection{HER1/EGFR in Proliferation and Survival of Keratinocytes}

The HER1/EGFR-dependent signaling in keratinocytes denotes a pathway that controls proliferation of this 
epidermal cell type. As a ligand, EGF was identified by its activity in the proliferation and maturation of the epidermal tissue. Similarly, the production of HER1/EGFR knock-out mice exhibiting no expression of the HER1/EGFR revealed, among other profound modifications, alterations in the characteristics of the epidermis, demonstrating the crucial tissue control afforded by the interaction between EGF and HER1/EGFR in keratinocytes [9, 10]. In cell culture, the requirement for EGF in cultures of keratinocytes has been illustrated in both serum-containing model [11] and in serum-free conditions [12]. Moreover, the identification of culture conditions that allow autocrine growth of human keratinocytes have depicted amphiregulin as a secreted growth factor in proliferating cultures of keratinocytes, but also demonstrated the critical ligand-binding on the HER1/EGFR in order to maintain the proliferation of this cell type in absence of any added growth factor in culture medium [13-15]. However, whereas the addition of EGF could not rescue keratinocytes from growth-arrest in confluent cultures when the cells are committed towards terminal differentiation $[16,17]$, the addition of EGF to those keratinocytes was nonetheless still able to suppress the gene expression of early differentiation markers [16].

Downstream to the activation of the tyrosine kinase activity in cytoplasmic domain of HER1/EGFR, adapting intermediates transduce the signal to Ras and mitogenactivated protein kinase (MAPK) pathways. Particularly, the HER1/EGFR-ERK1/2 MAPK pathway plays a crucial role in mediating the proliferative program in keratinocytes. Indeed, any interruption of the signaling induced by the autocrine growth factors will not only cause the arrest of keratinocyte proliferation, it will also induce irreversible commitment towards terminal differentiation [18]. This observation illustrates the significance of HER1/EGFR in keratinocyte cycle progression and thus proliferation [19, 20]. Consequently, a blockade of HER1/EGFR-dependent signaling, either by antagonist antibodies or selective inhibitors of kinase activities, induces keratinocyte susceptibility to cell death, for instance after a stress-like UV exposure [21]. Contrarily, the activation of the HER1/EGFR and MAPK pathway protects keratinocytes against the cell death that results from culture in suspension [22].

Along with the classical signal transduction cascade involving the MAPK ERK1/2 that has been extensively studied in many cell types as well as in keratinocytes following HER1/EGFR activation [23], the phosphatidylinositol 3-kinase (Pi3K) recruitment and activation result, through the production of phosphoinositides, in the phosphorylation and activation of the critical serine-threonine in Akt, a kinase that plays pivotal role in cell survival [24]. In addition, the recruitment of phospholipase C gamma (PLC $\gamma$ ) by the activated HER1/EGFR receptor is directly responsible for the production of diacylglycerol (DAG), a molecule that recruits and activates the protein kinase $\mathrm{C}$ (PKC), another important epidermal signal transducer. Indeed, PKC has been exhaustively studied in keratinocytes and its role has been demonstrated in the regulation of keratinocyte proliferation, as well as in the control of epidermal differentiation [25].

\subsection{HER1/EGFR in Adhesion and Migration of Keratinocytes}

The HER1/EGFR is also involved in the regulation of keratinocyte adhesion and migration by its multiple interactions with the transmembrane adhesion proteins of the integrin family. Integrins are heterodimeric adhesive receptors.

In keratinocytes, integrins of the $\beta 1$ subtype organize focal adhesion complexes that transmit information as intracellular signals and whose activities are modulated by EGF-dependent signaling. For instance, Chen and coworkers have largely illustrated that EGF acts on $\beta 1$ integrins in order to enhance the cellular mobility of keratinocytes [26]. These observations can be correlated with the enhanced integrin expression during epidermal wound healing [27], but also with the positive role played by EGF, or more physiologically by ligands that bind the HER1/EGFR, on the repair mechanisms in the skin and epidermis [28].

A second subtype of integrin, the integrin $\alpha 6 \beta 4$, is particularly important in the physiology of epidermal keratinocytes where it concentrates in hemidesmosomes. It has been shown that signaling from integrin $\alpha 6 \beta 4$ functions through MAPK and is thus acting closely to the signaling induced by the activated HER1/EGFR [29]. Also, signaling from the HER1/EGFR through Src kinase family members was found to disrupt the function of $\alpha 6 \beta 4$ and lead to disassembly of hemidesmosomes and stimulation of keratinocyte migration [30]. Very interestingly, $\alpha 6 \beta 4$ is further linked to the mitogenic effect of EGF on keratinocyte by its compartmentalization into specialized plasma membrane called lipid rafts (that will be described below), suggesting that such domains are crucial for the normal control and function of keratinocytes [31]. Integrindependent phosphorylation of Tyr-845 in HER1/EGFR, a tyrosine located in the activation loop of the receptor, may occur through Src kinase activity and is a priming event for subsequent activation of the HER1/EGFR tyrosine kinase activity. This activity is required for the phosphorylation of the other tyrosine residues of the receptor [32].

Thus HER1/EGFR activity controls adhesion and migration of keratinocytes in collaboration with integrins that are normally expressed in the basal epidermal layer. Normal commitment of keratinocytes towards differentiation is accompanied by the inhibition of integrin expression, but an abnormal suprabasal expression of integrins is seen in psoriasis. Interestingly, this pathological situation can be experimentally mimicked by forcing the suprabasal expression of $\beta 1$ integrin in keratinocytes [33], or by the epidermal overexpression of amphiregulin [34], the HER1/EGFR ligand that allows autocrine growth of keratinocytes in serum-free culture conditions [14].

\subsection{HER1/EGFR in the Differentiation of Keratinocytes}

As already mentioned above, the treatment of keratinocytes with EGF initiates HER1/EGFR -dependent signaling and results in the suppression of the expression of certain markers of epidermal differentiation in keratinocytes, 
namely the suprabasal keratins 1 and $10[16,35]$. We have also reported a similar inhibition of the expression of keratins 1 and 10 when signaling is induced in keratinocytes by Heregulins that activate the receptors HER 2 and HER3 [2] or by treatment of keratinocytes with HB-EGF [36]. Altogether, these reports suggest that signaling through HER family rather inhibits some epidermal differentiation; however, a few reports have illustrated that high concentrations of EGF are able to induce the expression of particular markers of epidermal differentiation; especially the involucrin expression is enhanced after EGF or HB-EGF treatment [36, 37], decoupling EGF-dependent signaling from an automatic inhibitory action on the differentiation process.

The HER1/EGFR is located inside the plasma membrane on the cell surface. However the plasma membrane environment is currently considered as non homogenous and can differentially influence the activity of receptors embedded in the membrane. The next part of this review intends to characterize specialized plasma membrane microdomains.

\section{WHAT DO WE KNOW ABOUT LIPID RAFTS?}

Within the plasma membrane, the presence of different lipid species (glycerophospholipids, sphingolipids, sterols) is responsible for important variability in the degree of membrane ordering, creating zones where lipids such as cholesterol and sphingolipids are not distributed evenly but clustered into highly ordered membrane microdomains. This phenomenon allows formation of particular membrane areas that have been termed "detergent resistant membrane" (DRM), "liquid-ordered phase", or "detergent-insoluble glycolipid rich domains" (DIG), depending on the technique that has been used for the isolation or identification of such microdomains. However, even though the identifying techniques were different, researchers studying those microdomains realized that they were more or less working on similar materials, leading Simons and Ikonen to propose a new concept named "lipid rafts" [38]. Thus, according to available knowledge, lipid rafts were described as tightly packed and highly ordered domains inside the plasma membrane. The lipid rafts are containing high concentrations of specific lipids (sphingolipids and cholesterol) but are not devoid of proteins, localized as transmembrane proteins or covalently anchored on the outer or inner surfaces of the rafts [39]. Interestingly, enrichment of signaling molecules within the lipid rafts domains of the plasma membrane has led to the hypothesis that these structures are able to maintain preassembled complexes of signaling molecules, obviating the need to recruit each partner to the signaling structure, therefore increasing the speed of response to stimuli.

Consequently, lipid rafts have also been proposed as molecular platform important for signaling processes, despite the fact that a complete and precise definition was still lacking.

Recently, scientists with various backgrounds met in order to adopt a consensus about such a definition. This meeting led to the following definition: "Membrane rafts are small $(10-200 \mathrm{~nm})$, heterogeneous, highly dynamic, steroland sphingolipid-enriched domains that compartmentalize cellular processes. Small rafts can sometimes be stabilized to form larger platforms through protein-protein and proteinlipid interactions" [40]. However, this definition, while bringing precision about the fact that it can exist different kinds of raft, is still highly theoretical and provides no practical or experimental criteria for the identification of lipid rafts and their protein components inside the ocean membrane. Indeed, on one hand, no specific targeting sequence has been discovered yet in identified raft proteins (supposing that such sequence would exist), impeding any potential prediction of a localization of certain proteins inside lipid rafts, on the other hand the identification of proteins surely localized in lipid rafts remains highly difficult when using techniques such as proteomic or membrane fractionation on gradient alone. In addition, heterogeneity inside the rafts (both in size and composition) enhances the difficulty to precisely define such microdomains.

In regard of such a difficult definition of lipid rafts, the presence of caveolae in plasma membranes is an important possibility for confusion as caveolae share similar characteristics (resistance to detergent and buoyancy in sucrose gradient) with other kinds of lipid rafts. Of course, lipid rafts are flat portion of the plasma membrane, whereas caveolae form membrane invaginations due to the presence of caveolin-1. Despite this difference in morphology, the term "caveolae" is sometimes used synonymously with "lipid rafts". Finally, most biochemical/biophysical methods that enrich rafts also enrich caveolae. Consequently, "the" structural protein of caveolae, caveolin-1, is often assimilated to cholesterol-enriched regions, due to its ability to directly bind cholesterol [41], enhancing the confusion between caveolae and lipid rafts.

Since lipid rafts and caveolae $(100 \mathrm{~nm})$ are smaller than the resolution of light fluorescence microscopy $(+/-200 \mathrm{~nm})$, direct observation of lipid rafts does not provide unequivocal answer to the question of co-localisation. So lipid rafts are currently considered as spatiotemporally regulated dynamic lipid-dependent segregation of specific membrane components.

Recently, Yao et al. have discriminated caveolar membranes and non-caveolar lipid microdomains [42]. For their investigation, these authors used a modified four-step sucrose density gradient, allowing the partition of light lowdensity insoluble membrane domains and heavy low-density insoluble membrane domains and found that lipid rafts were more enriched in cholesterol than caveolae. In addition, the protein populations were different since caveolae seemed to contain cytoskeletal molecules, whereas lipid rafts contain molecules involved in signaling. Surprisingly, flotillin-1, that is considered to be more specific for lipid raft domains, was rather found in the caveolar portion of the gradient.

About the utilization of centrifugation gradients for the analysis of lipid rafts, it must be noticed that almost every laboratory working on this matter developed its own protocol, including or not the use of detergent. One of the characteristics of lipid rafts is precisely the resistance of those domains to disorganization by detergent. Lipid rafts are resistant to Triton X-100, but this detergent appears to cluster rafts, creating non pre-existing domains in the plasma membrane $[43,44]$. Moreover, there exist differences 
between rafts isolated in the presence of different detergents (Triton X-100, Brij 98, Brij 96, Tween 20) [45].

Other protocols using sonication of the cells in $\mathrm{pH} 11$ sodium carbonate buffer results in rafts fractions that are contaminated with other membranes. Methodology plays thus a key role in the outcome of any detergent solubilization experiments. This is why Macdonald and Pike have developed a detergent-free method for lipid raft purification [46].

Another experimental way to study the importance of lipid rafts in cell physiology is the disorganization of lipid rafts by mean of cholesterol extraction. Among the various molecules that can be used to deplete membrane cholesterol, methyl- $\beta$-cyclodextrin $(\mathrm{M} \beta \mathrm{CD})$ appears to be the more efficient and widely used tool for the study of lipid rafts. $\mathrm{M} \beta \mathrm{CD}$ is a membrane impermeable, small cyclic oligosaccharide with a hydrophobic core that selectively extracts cholesterol from the plasma membrane [47, 48]. Another widely used molecule is the polyene antibiotic filipin from Saccharomyces filipinensis. Filipin forms multimeric pack of cholesterol within the plasma membrane, inducing structural disorder in the plasma membrane [49, 50].

In conclusion, although the raft hypothesis is well recognized and accepted by use of gradient fractionation, the utilization of new techniques, including FRET, atomic-force microscopy or single-particle tracking experiments will provide clues that lipid rafts are more complex than stable, freely diffusing specialized membrane domains made up of highly ordered lipids and proteins [51].

\section{CONTROL OF THE HER1/EGFR BY LIPID RAFTS}

\subsection{Localization of HER1/EGFR in Lipid Rafts}

As we describe above in the first part of this review, HER1/EGFR activation and processing are crucial for the control of epidermal keratinocytes. Upon ligand binding, the HER1/EGFR is believed to move out of the raft portion of the plasma membrane, to form dimers and to be internalized within clathrin-coated pits as depicted in Fig. (1), (for a review, see [52]). Whether the activity of HER1/EGFR can be regulated by its localization inside membrane lipid rafts or outside of these specialized domains has been a matter of interest. The plasma membrane cholesterol appears to regulate freedom of the HER1/EGFR inside the membrane [53] and moreover, the activation of HER1/EGFR by a specific ligand can induce coalescence of membrane lipid rafts [54]. Thus, the localization of HER1/EGFR and other growth factor receptors in the plasma membrane has been largely studied. If most growth factor receptors localize to the caveolar part of the membrane, HER1/EGFR is a notable exception since more than half of the total HER1/EGFR population is apparently found in non-caveolar rafts [55] whilst smaller percentages are present in caveolae and clathrin-coated pits [56]. Yet previous data had initially localized HER1/EGFR in caveolae [57].

Different populations of HER1/EGFR may thus reside in various areas of the plasma membrane and these localizations may be linked to differences both in activation and internalization of the receptor. Indeed, accumulating evidences have suggested that internalization of the
HER1/EGFR is performed at the level of clathrin-coated pits, as well as at the level of non-coated membrane domains, maybe reflecting divergent processing of the receptor, depending on its localization in lipid rafts or not $[58,59]$.

\subsection{Activation of the HER1/EGFR Signaling Pathway Following Lipid Rafts Disruption}

Binding of the HER1/EGFR by its cognate ligands induces the activation of the intrinsic tyrosine kinase, ensuing receptor autophosphorylation and recruitment/ activation of the signal transduction machinery. Concurrently, ligand-activated receptors trigger their own endocytosis in order to extinguish signaling. Actually signaling takes place mostly inside lipid rafts, whereas endocytosis occurs mainly at the level of clathrin-coated pits where activated receptors are clustered for efficient internalization.

Several independent investigations have demonstrated that the receptor activity of the HER1/EGFR is significantly enhanced after the disruption of lipid rafts by cholesterol depletion using methyl- $\beta$-cyclodextrin. In addition, a concomitant increase in radiolabelled ligand-binding on the HER1/EGFR is observed after lipid rafts disruption $[55,60$, 61]. Such an increased binding is likely due to a better accessibility of the receptors for the ligand, maybe due to some flattening of clathrin-coated pits and caveolae that results from the incubation with methyl- $\beta$-cyclodextrin [6264] (Fig. 1).

Since HER1/EGFR is activated in a ligand-independent manner following cholesterol depletion, several different mechanisms likely responsible for this activation have been proposed. In the peculiar case of HER1/EGFR, lipid raft disorganization appears to unmask receptor at the cell surface, increasing the number of exposed HER1/EGFR to ligand without externalisation of an internal pool of the receptor [60]. Some clues concerning the activation of the HER1/EGFR following cholesterol depletion have been given [65], however we still do not know precisely what triggers this activation. Possibly Src kinases could be involved since Tyr- 845 on the HER1/EGFR is a site known to be phosphorylated by $\mathrm{pp} 60^{\text {src }}$ and its phosphorylation positioned in the activation loop of the kinase domain is associated with stimulated kinase activity of HER1/EGFR [66]. Indeed, Tyr-845 is found phosphorylated following lipid raft disruption [67], however an inhibition of the Src kinases is unable to totally impede HER1/EGFR activation [65], strongly suggesting that other mechanisms might be involved in ligand-independent HER1/EGFR activation. For instance, we could propose that some destabilisation of the receptor may occur once HER1/EGFR is localized outside lipid rafts due to an unusual molecular environment in the plasma membrane. This new environment might allow changes in structural conformation of the HER1/EGFR, leading to the exposure of the dimerization arm and torsion of the cytoplasmic tail. Also, an increased liberty for lateral movements of HER1/EGFR in the plasma membrane, or a local increase in size of the HER1/EGFR population might strongly enhance the frequency of receptors clustering and dimers formation. 


\section{+ligand}

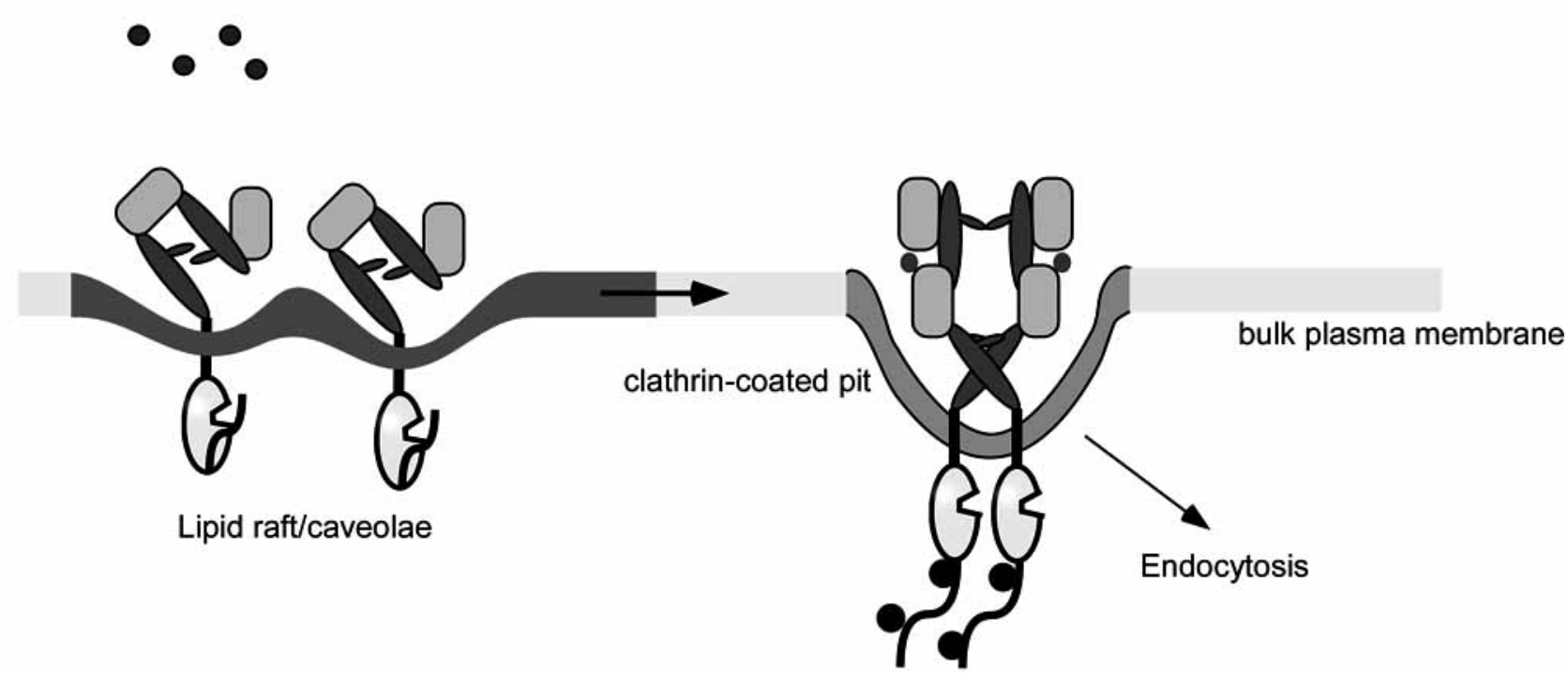

\section{$+\mathrm{M} \beta \mathrm{CD}$}
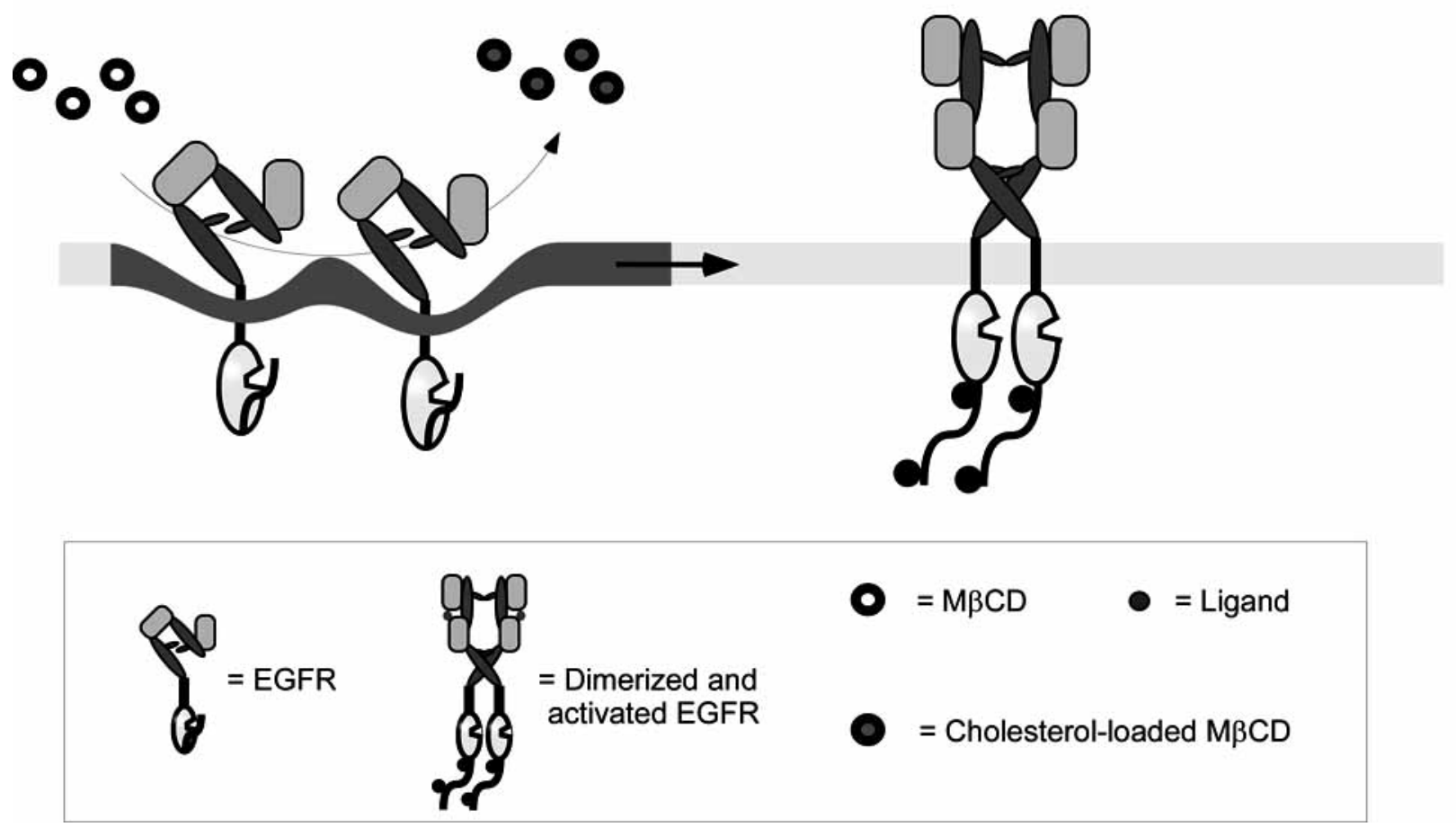

Fig. (1). Comparison between ligand-induced activation of the HER1/EGFR and its activation consecutive to the disruption of lipid rafts/caveolae. The HER1/EGFR is located in the lipid rafts/caveolae fraction of the plasma membrane. Upon stimulation with a ligand, the receptor forms dimers that migrate outside the lipid rafts/caveolae and are internalized in clathrin-coated pits. Depletion of membrane cholesterol induces flattening and disorganization of lipid rafts/caveolae, leading to dimers formation, but not to internalization of the HER1/EGFR.

\section{THE HER1/EGFR IN LR IN KERATINOCYTE}

The presence of lipid rafts in the plasma membrane of epidermal keratinocytes has been demonstrated and shown to rearrange during cell culture. As those microdomains were especially reported in transit amplifying keratinocytes [68], we postulated an involvement of lipid rafts in the regulation of phenotype in the epidermal cells. Indeed, a treatment of cultured human keratinocytes with methyl- $\beta$-cyclodextrin for 1 hour resulted in reduction of the total cholesterol content of 
cells, as attested by cholesterol assay or filipin staining [37], and the prolonged cholesterol depletion maintained by the use of lovastatin as an inhibitor of cholesterol synthesis resulted in a strongly altered expression of epidermal differentiation markers, revealed by suppressed expression of keratins 14 and 10, whilst the expression of involucrin was accelerated [37]. This alteration was mimicked by treatment of keratinocytes with EGF, suggesting that activation of HER1/EGFR could be responsible for those observations. However, although the HER1/EGFR was indeed phosphorylated in those conditions, the specific inhibition of the tyrosine kinase activity of the receptor with PD153035 did not impede the phenotype alteration obtained through cholesterol depletion. Rather, we found that the kinase activity of p38 MAPK was required for the observed alteration of phenotype [36, 37]. This alteration was further demonstrated as resulting in the transient over-expression of the heparin-binding EGF-like growth factor (HB-EGF), a phenomenon that also depended on the activation of the p38 MAPK and which may be responsible for inhibition of suprabasal keratins and upregulation of involucrin [36].

As previously described in several cell types, as well as in normal human keratinocytes [37], we also found the HER1/EGFR activated in a ligand-independent manner in immortalized HaCaT keratinocytes [69]. Here again, the activation of HER1/EGFR by cholesterol depletion was followed by phosphorylation of the ERK1/2 MAPK and p38 MAPK as in normal human keratinocytes [70].

Several points emerge from those studies. First, the program of epidermal differentiation in keratinocytes appears to be altered upon disruption of cholesterol-enriched domains in the plasma membrane. This is due to the activation of p38 MAPK. However, even though p38 MAPK emerge as a highly significant actor in the response developed by keratinocytes towards cholesterol depletion, no explanation of mechanisms responsible for its activation has been put forward so far. Second, in response to the disorganization of cholesterol-enriched domains, keratinocytes trigger two apparently opposing reactions: the late differentiation process seems started up because the expression of involucrin is upregulated, but at the same time, the specific HER1/EGFR ligand HB-EGF is produced and released in the cellular environment. Thus, cholesterolenriched domains (lipid rafts and caveolae, as no distinction between them can be made when using $\mathrm{M} \beta \mathrm{CD}$ ) are playing major roles in regulation of keratinocyte phenotype (Fig. 2).

Because a long-term incubation of keratinocytes with $\mathrm{M} \beta \mathrm{CD}$ is known to induce cell death, likely through apoptotic stimulation $[71,72]$, it is of interest to allow cells recovering from shorter ( 1 hour) cholesterol depletion and lipid raft disruption. Indeed, during recovery periods, keratinocytes restore normal signalization since HER1/EGFR and p38 MAPK phosphorylations disappear, although the cellular proliferation is unaffected [70]. Interestingly, and despite previous studies have shown the inability of cells to internalize HER1/EGFR during lipid raft disruption [73], we observed that during the recovery period of keratinocytes, a particular internalization of HER1/EGFR occurred [70]. This internalization was a phenomenon clearly dependent on the activity of p38 MAPK (Fig. 2), resembling a process recently described in HeLa cells submitted to stressing conditions $[74,75]$.

\section{$+\mathrm{M} \beta \mathrm{CD}$}

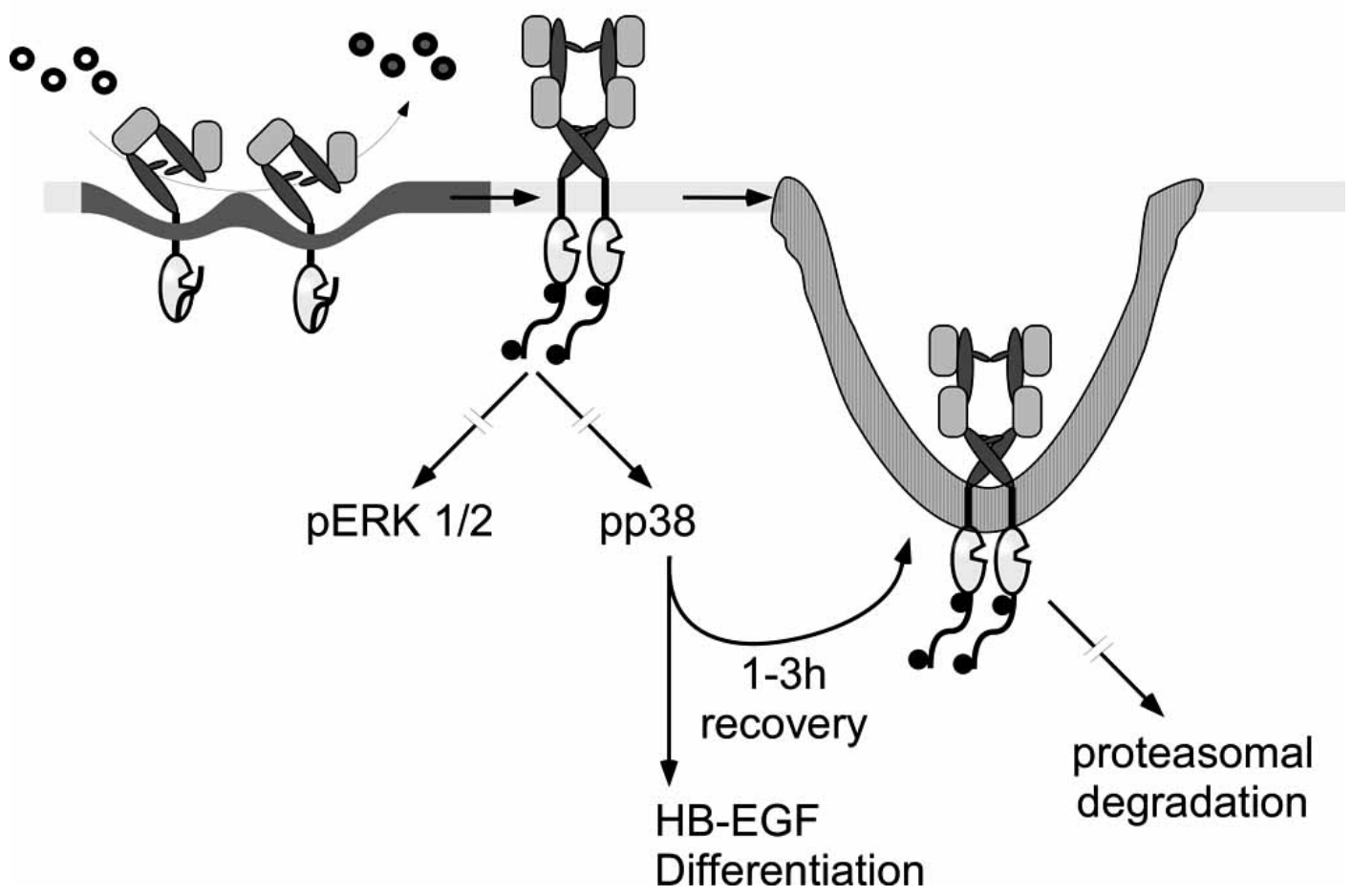

Fig. (2). Fate of the HER1/EGFR following disruption of lipid raft/caveolae. Upon cholesterol depletion by M $\beta C D$, the HER1/EGFR is activated and form dimers that subsequently activate ERK $1 / 2$ and p38 MAPK. During the recovery period, activated p38 MAPK induces keratinocytes differentiation [37], HB-EGF shedding [36] and HER1/EGFR internalization through yet undefined structures, leading to proteasomal degradation [69]. 
Hypothetically, such an internalization of the HER1/EGFR following cholesterol depletion and lipid raft disruption could be a protective measure taken by keratinocytes in order to prevent the stimulation of the receptor through ligandbinding (e.g. HB-EGF) during the stressful condition i.e. membrane fluidization, induced by cholesterol removal [76]. This hypothesis is supported on one hand by the observation that mechanical scratches in keratinocyte cultures induce activation and p38 MAPK-dependent internalization of the HER1/EGFR (unpublished data) and on the other hand by the detection of HB-EGF expression in stressful conditions such as wound healing, oxidative stress, and cholesterol depletion [36].

In summary, cholesterol-enriched domains in the plasma membrane of epidermal keratinocytes could be central platform managing cell signalization in this cell type. Lipid rafts and caveolae provide thus protection and alert signals towards neighboring cells after stress of the tissue by the mean of activation of the HER1/EGFR and consequently p38 MAPK pathway activation.

\section{CONCLUSION}

Keratinocytes physiology appears to be modulated by events interfering with the integrity/structure of the plasma membrane cholesterol-enriched domains commonly named lipid rafts and caveolae. Specially the regulation of the signaling through the HER1/EGFR appears to be highly regulated by its membrane molecular environment. As the HER1/EGFR represents maybe the most influent regulator in normal and pathological epidermal conditions, the control of its interactions within particular plasma membrane domains could represent a target for future investigations and therapeutics.

\section{ACKNOWLEDGEMENTS}

SL holds a fellowship from the FRIA, Belgium. This research received support from FRFC grant 2.4506.01 and FNRS grant 1.5.033.06F to YP.

\section{REFERENCES}

[1] Edwin F, Wiepz GJ, Singh R, et al. historical perspective of the EGF receptor and related systems. Methods Mol Biol 2006; 327: 124

[2] De Potter IY, Poumay Y, Squillace KA, et al. Human EGF receptor (HER) family and heregulin members are differentially expressed in epidermal keratinocytes and modulate differentiation. Exp Cell Res 2001; 271: 315-28.

[3] Nanney LB, Magid M, Stoscheck CM, et al. Comparison of epidermal growth factor binding and receptor distribution in normal human epidermis and epidermal appendages. J Invest Dermatol 1984; 83: 385-93.

[4] Nanney LB, Stoscheck CM, King LE Jr, et al. Immunolocalization of epidermal growth factor receptors in normal developing human skin. J Invest Dermatol 1990; 94: 742-8.

[5] Coffey RJ Jr, Derynck R, Wilcox JN, et al. Production and autoinduction of transforming growth factor-alpha in human keratinocytes. Nature 1987; 328: 817-20.

[6] Hashimoto K, Higashiyama S, Asada H, et al. Heparin-binding epidermal growth factor-like growth factor is an autocrine growth factor for human keratinocytes. J Biol Chem 1994; 269: 20060-6.

[7] Piepkorn M, Lo C, Plowman G. Amphiregulin-dependent proliferation of cultured human keratinocytes: autocrine growth, the effects of exogenous recombinant cytokine, and apparent requirement for heparin-like glycosaminoglycans. J Cell Physiol 1994; 159: 114-20.

[8] Poumay YG. The dumb ErbB receptor helps healing. J Invest Dermatol 2007; 127: 995-7.
[9] Sibilia M, Wagner EF. Strain-dependent epithelial defects in mice lacking the EGF receptor. Science 1995; 269: 234-8.

[10] Threadgill DW, Dlugosz AA, Hansen LA, et al. Targeted disruption of mouse EGF receptor: effect of genetic background on mutant phenotype. Science 1995; 269: 230-4.

[11] Rheinwald JG, Green H. Epidermal growth factor and the multiplication of cultured human epidermal keratinocytes. Nature 1977; 265: 421-4.

[12] Boyce ST, Ham RG. Calcium-regulated differentiation of normal human epidermal keratinocytes in chemically defined clonal culture and serum-free serial culture. J Invest Dermatol 1983 81(Suppl): 33s-40s.

[13] Cook PW, Mattox PA, Keeble WW, et al. A heparin sulfateregulated human keratinocyte autocrine factor is similar or identical to amphiregulin. Mol Cell Biol 1991; 11: 2547-57.

[14] Cook PW, Pittelkow MR, Shipley GD. Growth factor-independent proliferation of normal human neonatal keratinocytes: production of autocrine- and paracrine-acting mitogenic factors. J Cell Physiol 1991; 146: 277-89.

[15] Pittelkow MR, Cook PW, Shipley GD, et al. Autonomous growth of human keratinocytes requires epidermal growth factor receptor occupancy. Cell Growth Differ 1993; 4: 513-21.

[16] Poumay Y, Pittelkow MR. Cell density and culture factors regulate keratinocyte commitment to differentiation and expression of suprabasal K1/K10 keratins. J Invest Dermatol 1995; 104: 271-6.

[17] Wille JJ Jr, Pittelkow MR, Shipley GD, et al. Integrated control of growth and differentiation of normal human prokeratinocytes cultured in serum-free medium: clonal analyses, growth kinetics, and cell cycle studies. J Cell Physiol 1984; 121: 31-44.

[18] Peus D, Hamacher L, Pittelkow MR. EGF-receptor tyrosine kinase inhibition induces keratinocyte growth arrest and terminal differentiation. J Invest Dermatol 1997; 109: 751-6.

[19] Coffey RJ Jr, Sipes NJ, Bascom CC, et al. Growth modulation of mouse keratinocytes by transforming growth factors. Cancer Res 1988; 48: 1596-602

[20] Kobayashi T, Hashimoto K, Okumura H, et al. Endogenous EGFfamily growth factors are necessary for the progression from the G1 to S phase in human keratinocytes. J Invest Dermatol 1998; 111: 616-20.

[21] Jost M, Gasparro FP, Jensen PJ, et al. Keratinocyte apoptosis induced by ultraviolet B radiation and CD95 ligation -- differential protection through epidermal growth factor receptor activation and Bcl-x(L) expression. J Invest Dermatol 2001; 116: 860-6.

[22] Jost M, Huggett TM, Kari C, et al. Matrix-independent survival of human keratinocytes through an EGF receptor/MAPK-kinasedependent pathway. Mol Biol Cell 2001; 12: 1519-27.

[23] Schlessinger J. Cell signaling by receptor tyrosine kinases. Cell 2000; 103: 211-25.

[24] Song G, Ouyang G, Bao S. The activation of Akt/PKB signaling pathway and cell survival. J Cell Mol Med 2005; 9:59-71.

[25] Mitev V, Miteva L. Signal transduction in keratinocytes. Exp Dermatol 1999; 8: 96-108

[26] Chen JD, Kim JP, Zhang K, et al. Epidermal growth factor (EGF) promotes human keratinocyte locomotion on collagen by increasing the alpha 2 integrin subunit. Exp Cell Res 1993; 209: 216-23.

[27] Larjava H, Salo T, Haapasalmi K, et al. Expression of integrins and basement membrane components by wound keratinocytes. J Clin Invest 1993; 92: 1425-35.

[28] Hardwicke J, Schmaljohann D, Boyce D, et al. Epidermal growth factor therapy and wound healing--past, present and future perspectives. Surgeon 2008; 6: 172-7.

[29] Mainiero F, Pepe A, Yeon M, et al. The intracellular functions of alpha6beta4 integrin are regulated by EGF. J Cell Biol 1996; 134: 241-53.

[30] Mariotti A, Kedeshian PA, Dans M, et al. EGF-R signaling through Fyn kinase disrupts the function of integrin alpha6beta4 at hemidesmosomes: role in epithelial cell migration and carcinoma invasion. J Cell Biol 2001; 155: 447-58.

[31] Gagnoux-Palacios L, Dans M, van't Hof W, et al. Compartmentalization of integrin alpha6beta4 signaling in lipid rafts. J Cell Biol 2003; 162: 1189-96.

[32] Cabodi $\mathrm{S}$, Moro $\mathrm{L}$, Bergatto $\mathrm{E}$, et al. Integrin regulation of epidermal growth factor (EGF) receptor and of EGF-dependent responses. Biochem Soc Trans 2004; 32(Pt3): 438-42. 
[33] Carroll JM, Romero MR, Watt FM. Suprabasal integrin expression in the epidermis of transgenic mice results in developmental defects and a phenotype resembling psoriasis. Cell 1995; 83: 95768.

[34] Cook PW, Piepkorn M, Clegg CH, et al. Transgenic expression of the human amphiregulin gene induces a psoriasis-like phenotype. J Clin Invest 1997; 100: 2286-94.

[35] Drozdoff V, Pledger WJ. Commitment to differentiation and expression of early differentiation markers in murine keratinocytes in vitro are regulated independently of extracellular calcium concentrations. J Cell Biol 1993; 123: 909-19.

[36] Mathay C, Giltaire S, Minner F, et al. Heparin-binding EGF-like growth factor is induced by disruption of lipid rafts and oxidative stress in keratinocytes and participates in the epidermal response to cutaneous wounds. J Invest Dermatol 2008; 128: 717-27.

[37] Jans R, Atanasova G, Jadot M, et al. Cholesterol depletion upregulates involucrin expression in epidermal keratinocytes through activation of p38. J Invest Dermatol 2004; 123: 564-73.

[38] Simons K, Ikonen E. Functional rafts in cell membranes. Nature 1997; 387: 569-72.

[39] Munro S. Lipid rafts: elusive or illusive? Cell 2003; 115: 377-88.

[40] Pike LJ. Rafts defined: a report on the Keystone Symposium on Lipid Rafts and Cell Function. J Lipid Res 2006; 47: 1597-8.

[41] Murata M, Peranen J, Schreiner R, et al. VIP21/caveolin is a cholesterol-binding protein. Proc Natl Acad Sci USA 1995; 92: 10339-43.

[42] Yao Y, Hong S, Zhou H, et al. The differential protein and lipid compositions of noncaveolar lipid microdomains and caveolae. Cell Res 2009; 19: 497-506.

[43] Heerklotz H. Triton promotes domain formation in lipid raft mixtures. Biophys J 2002; 83: 2693-701.

[44] Mayor S, Maxfield FR. Insolubility and redistribution of GPIanchored proteins at the cell surface after detergent treatment. Mol Biol Cell 1995; 6: 929-44.

[45] Pike LJ, Han X, Gross RW. Epidermal growth factor receptors are localized to lipid rafts that contain a balance of inner and outer leaflet lipids: a shotgun lipidomics study. J Biol Chem 2005; 280: 26796-804.

[46] Macdonald JL, Pike LJ. A simplified method for the preparation of detergent-free lipid rafts. J Lipid Res 2005; 46: 1061-7.

[47] Kilsdonk EP, Yancey PG, Stoudt GW, et al. Cellular cholesterol efflux mediated by cyclodextrins. J Biol Chem 1995; 270: 17250-6.

[48] Ohtani Y, Irie T, Uekama K, et al. Differential effects of alpha-, beta- and gamma-cyclodextrins on human erythrocytes. Eur J Biochem 1989; 186: 17-22.

[49] Bolard J. How do the polyene macrolide antibiotics affect the cellular membrane properties? Biochim Biophys Acta 1986; 864: 257-304.

[50] Robinson JM, Karnovsky MJ. Evaluation of the polyene antibiotic filipin as a cytochemical probe for membrane cholesterol. J Histochem Cytochem 1980; 28: 161-8.

[51] Hancock JF. Lipid rafts: contentious only from simplistic standpoints. Nat Rev Mol Cell Biol 2006; 7: 456-62.

[52] Warren CM, Landgraf R. Signaling through ERBB receptors: multiple layers of diversity and control. Cell Signal 2006; 18: 92333.

[53] Orr G, Hu D, Ozcelik S, et al. Cholesterol dictates the freedom of EGF receptors and HER2 in the plane of the membrane. Biophys J 2005; 89: 1362-73.

[54] Hofman EG, Ruonala MO, Bader AN, et al. EGF induces coalescence of different lipid rafts. J Cell Sci 2008; 121(Pt 15): 2519-28.

[55] Ringerike T, Blystad FD, Levy FO, et al. Cholesterol is important in control of EGF receptor kinase activity but EGF receptors are not concentrated in caveolae. J Cell Sci 2002; 115(Pt 6): 1331-40.
[56] Keating E, Nohe A, Petersen NO. Studies of distribution, location and dynamic properties of EGFR on the cell surface measured by image correlation spectroscopy. Eur Biophys J 2008; 37: 469-81.

[57] Mineo C, Gill GN, Anderson RG. Regulated migration of epidermal growth factor receptor from caveolae. J Biol Chem 1999; 274: 30636-43.

[58] Puri C, Tosoni D, Comai R, et al. Relationships between EGFR signaling-competent and endocytosis-competent membrane microdomains. Mol Biol Cell 2005; 16: 2704-18.

[59] Sigismund S, Woelk T, Puri C, et al. Clathrin-independent endocytosis of ubiquitinated cargos. Proc Natl Acad Sci USA 2005; 102: 2760-5.

[60] Pike LJ, Casey L. Cholesterol levels modulate EGF receptormediated signaling by altering receptor function and trafficking. Biochemistry 2002; 41: 10315-22.

[61] Roepstorff K, Thomsen P, Sandvig K, et al. Sequestration of epidermal growth factor receptors in non-caveolar lipid rafts inhibits ligand binding. J Biol Chem 2002; 277: 18954-60.

[62] Rodal SK, Skretting G, Garred O, et al. Extraction of cholesterol with methyl-beta-cyclodextrin perturbs formation of clathrincoated endocytic vesicles. Mol Biol Cell 1999; 10: 961-74.

[63] Rothberg KG, Heuser JE, Donzell WC, et al. Caveolin, a protein component of caveolae membrane coats. Cell 1992; 68: 673-82.

[64] Westermann M, Steiniger F, Richter W. Belt-like localisation of caveolin in deep caveolae and its re-distribution after cholesterol depletion. Histochem Cell Biol 2005; 123: 613-20.

[65] Chen X, Resh MD. Cholesterol depletion from the plasma membrane triggers ligand-independent activation of the epidermal growth factor receptor. J Biol Chem 2002; 277: 49631-7.

[66] Biscardi JS, Maa MC, Tice DA, et al. c-Src-mediated phosphorylation of the epidermal growth factor receptor on Tyr845 and Tyr1101 is associated with modulation of receptor function. $\mathrm{J}$ Biol Chem 1999; 274: 8335-43.

[67] Westover EJ, Covey DF, Brockman HL, et al. Cholesterol depletion results in site-specific increases in epidermal growth factor receptor phosphorylation due to membrane level effects. Studies with cholesterol enantiomers. J Biol Chem 2003; 278: 51125-33.

[68] Gniadecki R, Christoffersen N, Wulf HC. Cholesterol-rich plasma membrane domains (lipid rafts) in keratinocytes: importance in the baseline and UVA-induced generation of reactive oxygen species. J Invest Dermatol 2002; 118: 582-8.

[69] Lambert S, Vind-Kezunovic D, Karvinen S, et al. Ligandindependent activation of the EGFR by lipid raft disruption. $\mathrm{J}$ Invest Dermatol 2006; 126: 954-62.

[70] Lambert S, Ameels H, Gniadecki R, et al. Internalization of EGF receptor following lipid rafts disruption in keratinocytes is delayed and dependent on p38 MAPK activation. J Cell Physiol 2008; 217: 834-45.

[71] Bang B, Gniadecki R, Gajkowska B. Disruption of lipid rafts causes apoptotic cell death in HaCaT keratinocytes. Exp Dermatol 2005; 14: 266-72.

[72] Gniadecki R. Depletion of membrane cholesterol causes ligandindependent activation of Fas and apoptosis. Biochem Biophys Res Commun 2004; 320: 165-9.

[73] He YY, Huang JL, Chignell CF. Cleavage of epidermal growth factor receptor by caspase during apoptosis is independent of its internalization. Oncogene 2006; 25: 1521-31.

[74] Vergarajauregui S, San Miguel A, Puertollano R. Activation of p38 mitogen-activated protein kinase promotes epidermal growth factor receptor internalization. Traffic 2006; 7: 686-98.

[75] Zwang Y, Yarden Y. p38 MAP kinase mediates stress-induced internalization of EGFR: implications for cancer chemotherapy. EMBO J 2006; 25: 4195-206.

[76] Balogh G, Horvath I, Nagy E, et al. The hyperfluidization of mammalian cell membranes acts as a signal to initiate the heat shock protein response. FEBS J 2005; 272: 6077-86. 